\title{
Autoantibodies to thyroid peroxidase in patients with type 1 diabetes in Taiwan
}

\author{
Ching-Chung Chang, Chien-Ning Huang and Lee-Ming Chuang \\ Department of Internal Medicine, National Taiwan University, Taipei, Taiwan \\ (Correspondence should be addressed to Lee-Ming Chuang, Department of Internal Medicine, National Taiwan University Hospital, \\ 7 Chung-Shan South Road, Taipei, Taiwan)
}

\begin{abstract}
Objective: Type 1 diabetes mellitus is frequently associated with autoimmune thyroid disease (ATD). Genetic susceptibility to autoantibody formation in association with ATD and type 1 diabetes mellitus has been described with varying frequencies, but there is still debate about the situation in the Chinese population. We have, therefore, investigated the prevalence of anti-thyroid peroxidase (anti-TPO) in type 1 diabetic patients, and compared the effect of anti-glutamate decarboxylase (anti-GAD) on the thyroid autoimmunity in patients with type 1 diabetes mellitus in Taiwan.

Subjects and methods: Two hundred and forty-three subjects with type 1 diabetes mellitus and seventy unrelated normal controls were recruited for the detection of anti-TPO. Two hundred and seventeen sera from two hundred and forty-three type 1 diabetic patients were tested for anti-GAD. RIA and immunoprecipitation were used for anti-TPO and anti-GAD detection respectively.

Results: The intra-assay and interassay coefficients of variation of anti-TPO detected by the RIA method ranged from $5.5 \%$ to $11.1 \%$. Among 243 type 1 diabetic patients, $53(21.8 \%)$ were positive for antiTPO. Compared with those without thyroid autoimmunity, there was a female preponderance for the type 1 diabetic patients with thyroid autoimmunity (female:male, 99:91 vs 37:16 respectively). Among the type 1 diabetic patients with thyroid autoimmunity, anti-TPO tended to occur in those of older age or with long-standing disease. The frequency of anti-GAD was $45.6 \%$ (99 of 217), without gender preponderance (males:females, $18.0 \%$ vs $27.6 \%$ ). Compared with those with negative antiGAD, no significant difference of anti-TPO positivity for the type 1 diabetic patients with positive antiGAD was found.

Conclusion: Our data indicated that the RIA method for anti-TPO detection is sensitive and precise for routine clinical use. The presence of anti-TPO in $21.8 \%$ of our type 1 diabetic patients confirmed the strong association of ATD and type 1 diabetes mellitus without ethnic differences. The absence of correlation between anti-TPO and anti-GAD in our type 1 diabetic patients suggested genetic heterogeneity in the role of autoimmunity of type 1 diabetes mellitus and ATD among races.
\end{abstract}

European Journal of Endocrinology 139 44-48

\section{Introduction}

The association of type 1 diabetes mellitus with autoimmune thyroid disease (ATD) has been well documented in many populations (1-7). The occurrence of thyroid autoantibodies against microsomes (AMA) and thyroglobulin (ATA), frequently seen in Hashimoto's thyroiditis and Graves' disease, has been reported in type 1 diabetes mellitus with varying frequency (2, 6-8). However, AMA tends to correlate better with thyroid dysfunction than does ATA (9). Although most type 1 diabetic patients are clinically euthyroid, screening for ATD by measuring AMA levels has been recommended as there is a risk of developing future thyroid dysfunction $(2,7)$. Recently, detection of antibodies against thyroid peroxidase (TPO), a major antigen for microsomal autoantibody, appears to obviate the need for AMA and ATA measurements because of the improvement in specificity and sensitivity of the method (10).

The prevalence of antibodies to islet-cell (ICA) and glutamate decarboxylase (anti-GAD) is significantly increased in patients with ATD compared with that of unaffected controls $(11,12)$. Genetic susceptibility of autoantibody formation associated with ATD and type 1 diabetes mellitus has been described in the literature (12-15); however, there is still debate about the situation in the Chinese population $(7,16-18)$. In the present study, we aimed to determine the frequency and titers of anti-TPO in Taiwanese patients with type 1 diabetes mellitus, and to compare the frequency of antiTPO in the presence or absence of anti-GAD. 


\section{Subjects, materials and methods}

\section{Subjects}

Sera were collected from 243 subjects with type 1 diabetes mellitus and 70 unrelated normal controls after informed consent was obtained. All the individuals recruited in this study were Han Chinese living in northern Taiwan. The type 1 diabetic patients were recruited from the Diabetic Clinic of the National Taiwan University Hospital, Taipei in the period 1990-1991 ( $n=78$, subset 1$)$, and in the period 1994-1995 ( $n=165$, subset 2 ). The mean age of type 1 diabetic subjects was 17.8 (range 6-46) years, and the diagnosis of type 1 diabetes mellitus was based on the published criteria (19) with a typical clinical history of diabetic ketoacidosis and a requirement for insulin (20). The control subjects were recruited from those who visited the hospital for general health examination. All the subjects were clinically euthyroid.

\section{Thyroid peroxidase antibody}

Autoantibodies to thyroid peroxidase (anti-TPO) were measured by radioimmunoassay (TPO Antibodies Radioimmunoassay Kit, Sorin Biomedica, Saluggio, Italy). The assay is based on the competition between anti-TPO in patient's sera and the solid-phase Fab to TPO (mouse monoclonal) for the fixed and limited number of labeled TPO binding sites. After incubation, the amount of antiTPO present in the patient's sera is inversely related to that of labeled TPO bound to the solid phase. The results of anti-TPO were obtained according to a calibration curve. The upper normal limit, determined as the mean+2s.D. of the data obtained from the sera of the controls in the study, was 11.4 arbitrary units (AU)/ml for anti-TPO, in agreement with the reference range suggested by the manufacturer $(<10 \mathrm{AU} / \mathrm{ml})$. The intraand interassay coefficients of variation of the anti-TPO were calculated for 5 assays. The anti-TPO titers were also compared with AMA titers determined by passive hemagglutination (PH) assays (Fujirebio, Tokyo, Japan) in the sera of patients of subset 1 as previously reported (8).

\section{Antibodies to glutamate decarboxylase (anti-GAD)}

The presence of anti-GAD antibodies was tested in 217 samples from the type 1 diabetic patients with an immunoprecipitation method (21). Briefly, in vitro transcription of a plasmid DNA containing human $\mathrm{GAD}_{65}$ sequences (provided by Dr Dyrberg at Novo Nordisk A/S Industry, Denmark) and translation with $\left[{ }^{35} \mathrm{~S}\right]$ meth-ionine labeling was performed according to the manufacturer's instructions (TNT ${ }^{\mathrm{TM}} \mathrm{T} 7$ Coupled Reticulocyte Lysate System, Promega, Madison, WI, USA). Subsequently, $10 \mu \mathrm{l}$ serum were incubated with the labeled $\mathrm{GAD}_{65}$ overnight at $4^{\circ} \mathrm{C}$. After adsorption to protein A-sepharose beads, the antibody-bound fraction of labeled $\mathrm{GAD}_{65}$ was eluted and separated by polyacrylamide gel electrophoresis, and then autoradiographed. The band was deemed to be positive if the intensity of the band area exceeded the mean+2s.D. of the values from healthy controls.

\section{Statistical analysis}

Comparison between means was performed by Student's $t$-test and comparison between frequencies was carried out by chi-squared test. A $P$ value of 0.05 or less was interpreted as significant for the analysis.

\section{Results}

\section{Intra-assay and interassay variation of anti-TPO}

The intra-assay coefficients of variation of the anti-TPO assay were $5.5 \%$ at a mean value of $2.7 \mathrm{AU} / \mathrm{ml}(n=5)$; $6.7 \%$ at $77.3 \mathrm{AU} / \mathrm{ml}(n=5)$ and $6.7 \%$ at $768.9 \mathrm{AU} / \mathrm{ml}$ $(n=5)$. The interassay coefficients of variation were $6.9 \%$ at a mean value of $3.9 \mathrm{AU} / \mathrm{ml}(n=5) ; 6.0 \%$ at $54.9 \mathrm{AU} / \mathrm{ml}(n=5)$ and $11.1 \%$ at $370.1 \mathrm{AU} / \mathrm{ml}(n=5)$.

\section{Comparison of anti-TPO and AMA}

The sera of 78 subjects of subset 1 were available for analysis (8). There was a highly significant positive correlation $(r=0.889, P<0.001)$ between the titers of anti-TPO determined by RIA and AMA determined by $\mathrm{PH}$.

\section{Demographic characteristics among type 1 diabetic patients and thyroid autoimmunity}

Anti-thyroid peroxidase antibodies were detected in 53 $(21.8 \%)$ out of 243 patients and $2(2.9 \%)$ out of 70

Table 1 Frequencies and titers of anti-TPO in subjects with type 1 diabetes and normal controls. Data are shown as number (percentage).

\begin{tabular}{lcrrrr}
\hline & & \multicolumn{4}{c}{ Anti-TPO titers (AU/ml) } \\
\cline { 3 - 6 } Subjects & $\boldsymbol{n}$ & $<11.4$ & $11.4-100$ & $100-200$ & $200-500$ \\
\hline Type 1 diabetes & 243 & $190(78.2)$ & $20(8.2)$ & $13(5.3)$ & $12(5.0)$ \\
Normal & 70 & $68(97.1)$ & $2(2.9)$ & 0 & 0 \\
\hline
\end{tabular}


Table 2 Demographic characteristics of type 1 diabetic patients positive or negative for anti-TPO. Data are presented as means \pm S.D.

\begin{tabular}{lccc}
\hline & Positive & Negative & $\boldsymbol{P}$ value \\
\hline Number of patients & 53 & 190 & \\
Male:female & $16: 37$ & $91: 99$ & $<0.05^{*}$ \\
Age at diagnosis (years) & $13.4 \pm 7.8$ & $10.4 \pm 7.3$ & $\mathrm{NS}$ \\
Duration (years) & $7.8 \pm 6.2$ & $6.6 \pm 4.4$ & $\mathrm{NS}$ \\
BMl & $19.5 \pm 3.1$ & $19.6 \pm 3.0$ & $\mathrm{NS}$ \\
\hline
\end{tabular}

${ }^{*}$ By $\chi^{2}$-test.

NS, not significant.

normal controls. None of these subjects had any symptoms of thyroid disease. The distribution of antiTPO titers and positive frequencies of type 1 diabetic patients were variable as shown in Table 1 . The presence of significant anti-TPO titers $(>11.5 \mathrm{AU} / \mathrm{ml})$ was thought to indicate thyroid autoimmunity. As shown in Table 2, 16 out of 107 males $(15.0 \%)$ and 37 out of 136 females $(27.2 \%)$ had thyroid autoimmunity. A female predominance was noted in the cases with thyroid autoimmunity $(P<0.05)$. When patients were subdivided into those who had had the disease for a short duration (duration less than 1 year) and those of long-standing (more than 1 year) disease, this association of female predominance was also true for the group with long-standing type 1 diabetes mellitus (male: female $13.8 \%: 29.3 \% ; \quad P<0.01$ ), but not for the shorter-duration group (Table 3 ). There was a significantly higher frequency of anti-TPO positivity in older than in younger groups $(43.8 \%$ in the age group more than 25 years, $27.2 \%$ in the age group of 10 to 25 years, $15.6 \%$ in the age group less than 10 years; $\left.\chi^{2}=9.5, P<0.01\right)$. Except for the difference mentioned above, there was no remarkable difference in the body mass index (BMI).

\section{Anti-TPO and anti-GAD in type 1 diabetes mellitus}

The frequency of anti-GAD was $45.6 \%$ (99 out of 217), and no difference between males and females $(18.0 \%$ vs $27.6 \%)$ was found. The occurrence of positivity of antiTPO in the presence of anti-GAD was $23.2 \%$ (23 out of
99 subjects positive for anti-GAD). This difference was not statistically significant compared with $22.0 \%$ (26 of 118) among cases negative for anti-GAD. There was also no significant difference in patients with positive anti-GAD among those with shorter or long-standing duration of disease as shown in Table 3.

\section{Discussion}

Type 1 diabetes mellitus is strongly associated with other organ-specific diseases such as ATD, pernicious anemia, and idiopathic adrenal insufficiency $(1,4,22$, 23), while ATD has been reported to be the most common coexisting autoimmune disease with type 1 diabetes mellitus (24). There is also an increased prevalence of thyroid antibodies in type 1 diabetic patients with ATD $(2,3,7,13)$. The reasons for the increased frequency remain obscure. It was thought to result from a generally increased propensity to react against certain antigens, or from a genetically impaired ability to acquire tolerance to some autoantigens, or perhaps from certain common antigens present in the tissues prone to autoimmune disease (25). Among the thyroid autoantibodies, anti-TPO autoantibody, in addition to AMA and ATA, has recently become available for the determination of thyroid autoimmunity $(10,26)$. Anti-TPO assayed by monoclonal antibodyassisted RIA appears to be a more sensitive and specific marker for ATD than the conventional PH method (26). In the present study we have found an excellent correlation between AMA measured by $\mathrm{PH}$ and antiTPO activity measured by RIA in sera $(r=0.889 ; P<$ $0.001)$, in accordance with a previous report (27). The intra-assay coefficients of variation of the anti-TPO assay ranged from $5.5 \%$ to $6.7 \%$, while the interassay coefficients of variation ranged from $6.0 \%$ to $11.1 \%$ at different anti-TPO titers $(n=5)$, also indicating that the RIA measurement utilized in our study is a precise method of quantifying anti-TPO.

The prevalence of AMA in type 1 diabetic patients varied from $11.9 \%$ to $22 \%(2,7,28)$. As for the Chinese population, Tsai and Lee (7) and Chuang et al. (8) reported that $22 \%$ and $27.7 \%$ respectively of type 1 diabetic patients were positive for AMA, while Wong reported an absence of thyroid autoimmunity in Chinese children with type 1 diabetes mellitus (17).

Table 3 Prevalence of anti-TPO and anti-GAD in type 1 diabetic patients according to sex and duration of disease. Data are shown as number (percentage).

\begin{tabular}{|c|c|c|c|c|c|c|c|c|}
\hline \multirow[b]{2}{*}{ Antibody (+) } & \multicolumn{4}{|c|}{ Duration < 1 year } & \multicolumn{4}{|c|}{ Duration $>1$ year } \\
\hline & $\begin{array}{c}\text { Total } \\
(n=14)\end{array}$ & $\begin{array}{c}\text { Male } \\
(n=7)\end{array}$ & $\begin{array}{l}\text { Female } \\
(n=7)\end{array}$ & $P$ value & $\begin{array}{c}\text { Total } \\
(n=203)\end{array}$ & $\begin{array}{c}\text { Male } \\
(n=87)\end{array}$ & $\begin{array}{c}\text { Female } \\
(n=116)\end{array}$ & $P$ value \\
\hline $\begin{array}{l}\text { Anti-TPO } \\
\text { Anti-GAD }\end{array}$ & $\begin{array}{l}3 \\
8\end{array}$ & $\begin{array}{l}2(28.6) \\
4(57.1)\end{array}$ & $\begin{array}{l}1(14.3) \\
4(57.1)\end{array}$ & $\begin{array}{l}\text { NS } \\
\text { NS }\end{array}$ & $\begin{array}{l}46 \\
91\end{array}$ & $\begin{array}{l}12(13.8) \\
35(402)\end{array}$ & $\begin{array}{l}34(29.3) \\
56(48.3)\end{array}$ & $\begin{array}{c}<0.01 \\
\text { NS }\end{array}$ \\
\hline
\end{tabular}

NS, Not significant. 
Moreover, $10 \%$ to $24 \%$ of type 1 diabetic patients who were clinically euthyroid have been reported to be positive for anti-TPO $(14,15,29)$. From the sera of 243 type 1 diabetic patients in the present study, 53 (21.8\%) were positive for anti-TPO. The data confirmed the reports of Chuang and Tsai and their coworkers, and were in accordance with previous reports, indicating no ethnic difference in the association of ATD and Chinese type 1 diabetes mellitus.

High titers of anti-TPO were highly suggestive of ATD, and correlated well with thyroid dysfunction (30). The positive anti-TPO titers in our type 1 diabetic patients showed wide distribution. Among the patients with positive anti-TPO, the anti-TPO titers were mostly below $500 \mathrm{AU} / \mathrm{ml}(84.9 \% ; 45$ out of 53$)$, whereas only $15.1 \%$ of patients had titers greater than $500 \mathrm{AU} / \mathrm{ml}$ (Table 1). Although there was no apparent thyroid disease in the tested subjects at the time of investigation, further thyroid function evaluation should be regularly followed up because of the high risk of development of thyroid dysfunction in the future.

It is well known that organ-specific endocrine autoimmunity develops more frequently in women, including type 1 diabetes mellitus with thyroid autoimmunity (8). The production of anti-TPO is inheritable in an autosomal fashion in women but not in men (31). In our study, female preponderance with a male/female ratio of $16 / 37(P<0.05)$ was also found in the type 1 diabetic patients with thyroid autoimmunity. The association was also valid for the subgroup of longstanding type 1 diabetic patients, but not for the shorter-duration group. We also found a tendency for anti-TPO to occur with increasing age, in agreement with the report by Verge et al. (14). Taken together, a subgroup of type 1 diabetic patients associated with thyroid autoimmunity might present in the female patients of older age or with long-standing disease. The gene or other genetic predisposing factors responsible for this interesting phenomenon need to be investigated further. As for human leukocyte antigen (HLA) genes, we have previously identified that HLA DR3 and DR4 are associated with the susceptibility to type 1 diabetes mellitus regardless of thyroid autoimmunity in a Taiwanese population (8). With similar analysis in the present study, there was no difference in the HLA DR typing between the type 1 diabetic patients with or without positivity of anti-TPO (data not shown).

Varying frequencies of anti-GAD have been reported ranging from $25 \%$ to $70 \%(14,32-34)$, compared with a frequency of $45.6 \%$ (99 out of 217) in our study. In contrast to previous reports describing a higher frequency of anti-GAD in females compared with males, we failed to show a difference between the two sexes. There was also no difference in the occurrence of positive anti-GAD among patients with different duration of disease and different age at diagnosis. In view of the association of anti-GAD and type 1 diabetes mellitus with thyroid autoimmunity, Kawasaki et al. demonstrated that high levels of anti-GAD were present in type 1 diabetic patients with ATD (12). Martino et al. (32) revealed a significantly higher frequency of anti-TPO among anti-GAD positive than among anti-GAD negative adults with newly-diagnosed type 1 diabetes mellitus, whereas Verge et al. showed a similar association but without statistical significance (14). In the present study, we could not find any correlation between the presence of anti-TPO and anti-GAD. This may reflect the ethnic difference in the role of anti-TPO and anti-GAD immunity for type 1 diabetes mellitus with thyroid autoimmunity.

In conclusion, the utilization of a monoclonal antibodyassisted RIA for detection of anti-TPO has proved to be a sensitive and precise method for clinical routine use. The presence of anti-TPO in $21.8 \%$ of our patients with type 1 diabetes prompts the necessity for further thyroid function evaluation. The absence of a correlation between anti-TPO and anti-GAD in the Taiwan type 1 diabetic patients indicates an ethnic difference or genetic heterogeneity in the roles of autoimmunity of type 1 diabetes mellitus and ATD played by these two autoantibodies.

\section{Acknowledgements}

The authors thank Mr F S Lin for his technical assistance. This work was supported by a research fund from the National Taiwan University Hospital (to C C Chang) and a grant from the Diabetes Research Fund from the National Taiwan University Hospital (to L M Chuang).

\section{References}

1 Bottazzo GF, Mann JI, Thorogood M, Baum JD \& Doniach D. Autoimmunity in juvenile diabetes and their families. British Medical Journal 19782 165-168.

2 Riley W, MacLaren N, Zlezotte D, Spillar R \& Rosenbloom A. Thyroid autoimmunity in insulin-dependent diabetes mellitus: the case for routine screening. Journal of Pediatrics $198198350-$ 354.

3 Riley WJ, Winer A \& Goldstein D. Coincident presence of thyroidgastric autoimmunity at onset of type 1 (insulin-dependent) diabetes. Diabetologia 198324 418-421.

4 Betterle C, Zanette F, Pedini B, Presotto F, Rapp LB, Monciotti CM \& Rigon F. Clinical and subclinical organ-specific autoimmune manifestations in type 1 (insulin-dependent) diabetes patients and their first degree relatives. Diabetologia 198426 431-436.

5 Chikuba N, Akazawa S, Yamaguchi Y, Kawasaki E, Takino H, Takao Y, Maeda Y, Okuno S, Yamamoto H \& Yokota A. Type 1 (insulin-dependent) diabetes mellitus with coexisting autoimmune thyroid disease in Japan. Internal Medicine 199231 1076-1080.

6 Prina CLM, Weber G, Meschi F, Mora S, Bognetti E, Siragusa V \& Natale B. Prevalence of thyroid autoantibodies and thyroid autoimmune disease in diabetic children and adolescents. Diabetes Care $199417782-783$

7 Tsai WY \& Lee JS. Thyroid disease in Chinese children with type 1 diabetes. Diabetes Care $1993161314-1315$.

8 Chuang LM, Wu HP, Chang CC, Tsai WY, Chang HM, Tai TY \& Lin BJ. HLA DRB1/DQA1/DQB1 haplotype determines thyroid 
autoimmunity in patients with insulin-dependent diabetes mellitus. Clinical Endocrinology 199645 631-636.

9 Nordyke RA, Gilbert FI, Miyamoto LA \& Fleury KA. The superiority of antimicrosomal over antithyroglobulin antibodies for detecting Hashimoto's thyroiditis. Archives of Internal Medicine $1993153862-865$.

10 Feldt-Rasmussen Ulla. Analytical and clinical performance goals for testing autoantibodies to thyroperoxidase, thyroglobulin, and thyrotropin receptor. Clinical Chemistry 199642 160-163.

11 Yamaguchi Y, Chikuba N, Ueda Y, Yamamoto H, Yamasaki H, Nakanishi T, Akazawa S \& Nagataki S. Islet cell antibodies in patients with autoimmune disease. Diabetes $199140319-322$.

12 Kawasaki E, Takino H, Yano M, Uotani S, Matsumoto K, Takao Y, Yamaguchi Y, Akazawa S \& Nagataki S. Autoantibodies to glutamic acid decarboxylase in patients with type 1 diabetes and autoimmune thyroid disease. Diabetes 199443 80-86.

13 Landin-Olsson M, Karlsson A, Dahlquist G, Blom L, Lernmark A \& Sundkvist G. Islet cell and other organ-specific autoantibodies in all children developing type 1 (insulin-dependent) diabetes mellitus in Sweden during one year and in matched control children. Diabetologia 198932 387-395.

14 Verge CF, Howard NJ, Rowley MJ, Mackay IR, Zimmet PZ, Egan M, Hulinska H, Hulinsky I, Silvestrini RA, Kamath S, Sharp A Arundel T \& Silink M. Anti-glutamate decarboxylase and other antibodies at the onset of childhood type 1 diabetes: a populationbased study. Diabetologia 199437 1113-1120.

15 Abrams P, De Leeuw I \& Vertommen J. In new-onset insulindependent diabetic patients the presence of anti-thyroid peroxidase antibodies is associated with islet cell autoimmunity and the high risk haplotype HLA DQA1*0301-DQB1*0302. Diabetic Medicine 199613 415-419.

16 Tsai WY, Riley WJ, Lee JS \& Tsai WS. Islet cell and other organspecific autoantibodies in Chinese children with insulin-dependent diabetes mellitus. Journal of Formosan Medical Association 199089 969-971.

17 Wong GWK. Absence of thyroid disease in Chinese children with type 1 diabetes. Diabetes Care 199316 404-405.

18 Wong GWK. Autoimmune thyroid disease in Chinese children with type 1 diabetes. Diabetes Care 1993161315.

19 National Diabetes Data Group. Classification and diagnosis of diabetes mellitus and other categories of glucose intolerance. Diabetes 197928 1039-1057.

20 Chuang LM, Jou TS, Hu CY, Wu HP, Tsai WY, Lee JS, Hsieh RP, Chen KH, Tai TY \& Lin BJ. HLA-DQB1 codon 57 and IDDM in Chinese living in Taiwan. Diabetes Care 199417 863-868.

21 Chuang LM, Lin CY, Wu HP, Tsai WY, Tai TY \& Lin BJ. Anti-GAD 65 autoantibody in Taiwanese patients with insulin-dependent diabetes mellitus: effect of HLA on anti-GAD 65 positivity and clinical characteristics. Clinical Endocrinology 199747 455-461.

22 Beaven DW, Nelson DH, Renold AE \& Thorn GW. Diabetes mellitus and Addison's disease: a case report of eight patients and a review of 55 cases in the literature. New England Journal of Medicine 1959 $261443-454$.
23 Munichoodappa C \& Kozack GP. Diabetes mellitus and pernicious anemia. Diabetes 199019 719-723.

24 Mimura G, Kida K, Matsuura N, Toyoya T, Kitagawa T, Kobayashi T, Hibi I, Ikeda Y, Tsuchida I, Kuzuya H, Aono S, Doi K, Nishimukai H, Jinnouchi T \& Murakami K. Immunogenetics of early-onset insulin-dependent diabetes mellitus among Japanese: HLA, Gm, BF, GLO, and organ-specific autoantibodies: the JDS study. Diabetes Research and Clinical Practice 19908 253-262.

25 Norden G, Jensen E, Stilbo I, Bottazzo GF \& Lernmark A. B-cell function and islet cell and other organ-specific autoantibodies in relatives of insulin-dependent diabetic patients. Acta Medica Scandinavica 1983213 199-203.

26 Mariotti S, Caturegli P, Piccolo P, Barbesino G \& Pinchera A. Antithyroid peroxidase autoantibodies in thyroid diseases. Journal of Clinical Endocrinology and Metabolism 199071 661-669.

27 Mariotti S, Aneli S, Ruf J, Bechi R, Czarnocka B, Lombardi A, Carayon $\mathrm{P} \&$ \& Pinchera A. Comparison of serum thyroid microsomal and thyroid peroxidase autoantibodies in thyroid disease. Journal of Clinical Endocrinology and Metabolism 198765 987-993.

28 Kokkonen J, Kiuttu J, Mustonen A \& Rasanen O. Organ-specific antibodies in healthy and diabetic children and young adult. Acta Paediatrica Scandinavica 198271 223-226.

29 Doullay F, Ruf J, Codaccioni JL \& Carayon P. Prevalence of autoantibodies to thyroperoxidase in patients with various thyroid and autoimmune disease. Autoimmunity 19919 237244.

30 Vakeva A, Kontiainen S, Miettinen A, Schlenzka A \& Maenpaa J. Thyroid peroxidase antibodies in children with autoimmune thyroiditis. Journal of Clinical Pathology 199245 106-109.

31 Phillips D, Prentice L, Upadhyaya M, Lunt P, Chamberlain S, Roberts DF, McLachlan S \& Smith BR. Autosomal dominant inheritance of autoantibodies to thyroid peroxidase and thyroglobulin studies in families not selected for autoimmune thyroid disease. Journal of Clinical Endocrinology and Metabolism 199172 973-975.

32 Martino GV, Tappaz ML, Braghi S, Dozio N, Canal N, Pozza G, Bottazzo GF, Grimaldi LM \& Bosi E. Autoantibodies to glutamic acid decarboxylase (GAD) detected by an immuno-trapping enzyme activity assay: relation to insulin-dependent diabetes mellitus. Journal of Autoimmunity 1991 4 915-923.

33 DeAizpurua HJ, Harrison LC \& Cram DS. An ELISA for antibodies to recombinant glutamic acid decarboxylase in type 1 diabetes. Diabetes 199241 1182-1187.

34 Thivolet CH, Tappaz M, Durand A, Petersen J, Stefanutti A, Chatelain P, Vialettes B, Scherbaum W \& Orgiazzi J. Glutamic acid decarboxylase (GAD) autoantibodies are additional predictive markers of type 1 diabetes mellitus in high risk individuals. Diabetologia 199235 570-576.

Received 8 September 1997

Accepted 9 March 1998 